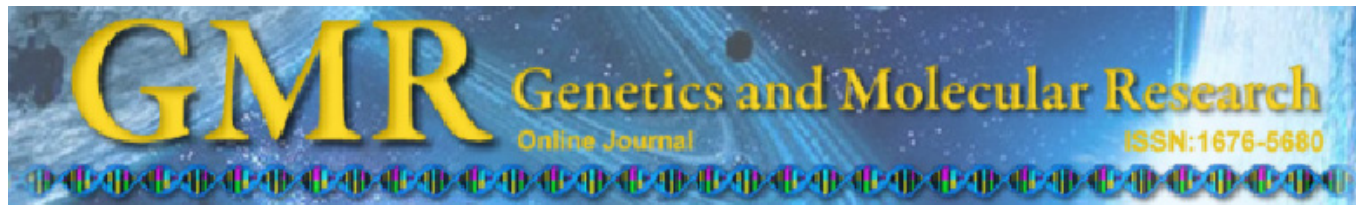

\title{
Resistance to lipopolysaccharide-induced endotoxic shock in heterozygous Zfp191 gene-knockout mice
}

\author{
R.L. Sun ${ }^{1,4}$, H.Y. Wang ${ }^{2,3}$, X.Y. Yang ${ }^{2,3}$, Z.J. Sheng ${ }^{3}$, L.M. Li ${ }^{2}$, L. Wang', \\ Z.G. Wang ${ }^{2}$ and J. Fei ${ }^{2,3}$ \\ ${ }^{1}$ Laboratory of Molecular Cell Biology, \\ Institute of Biochemistry and Cell Biology, \\ Shanghai Institutes for Biological Sciences, Chinese Academy of Sciences, \\ Shanghai, China \\ ${ }^{2}$ Shanghai Research Center for Model Organisms, Pu Dong, Shanghai, China \\ ${ }^{3}$ School of Life Science and Technology, Tongji University, Shanghai, China \\ ${ }^{4}$ Graduate School of Chinese Academy of Sciences, Beijing, China \\ Corresponding author. J. Fei \\ E-mail: jfei@tongji.edu.cn
}

Genet. Mol. Res. 10 (4): 3712-3721 (2011)

Received May 18, 2011

Accepted October 10, 2011

Published December 8, 2011

DOI http://dx.doi.org/10.4238/2011.December.8.6

\begin{abstract}
Zinc finger protein 191, ZNF24 and Zfp191 in both humans and mice belong to the SCAN domain subfamily of Krüppellike zinc finger transcription factors. Previous studies have suggested that Zfp191 is a pleiotropic factor involved in embryonic development, hematopoiesis and tumorigenesis. However, little is known about its target genes or its role in other physiological and pathological processes. We have identified the putative target genes of Zfp191, using an in silico genome-wide scan. Three hundred and fifty-five putative target genes were identified, which were enriched into the pathways of immune response according to the pathway analysis. These targets indicated that Zfp191 may function as a mediator of the immune response. This was verified in mice heterozygous for Zfp191 (Zfp191 $\left.{ }^{+/}\right)$using a lipopolysaccharide (LPS)-induced endotoxic shock model. After LPS injection, Zfp $191^{+/-}$mice produced significantly less IL-1 $\beta$ and IL-6 compared to wild-type mice and were resistant to LPS-induced endotoxic
\end{abstract}


shock. The loss of Zfp191 may suppress systemic inflammation by reducing these cytokine levels during LPS-induced endotoxic shock.

Key words: IL-1 $\beta$; IL-6; LPS; Zinc finger protein 191

\section{INTRODUCTION}

The mouse zinc finger protein 191 (Zfp191) belongs to the SCAN domain subfamily of Krüppel-like zinc finger transcription factors (Prost et al., 1999). These transcription factors contain a leucine-rich SCAN box and four consecutive $\mathrm{Cys}_{2} \mathrm{His}_{2}$ zinc fingers, indicating that they function in homo- or hetero-protein-protein recognition and protein-DNA/RNA interaction (Lu et al., 2003). ZNF24, the counterpart of Zfp191 in humans, shares a 94\% amino acid sequence identity with Zfp191 and is the most conserved SCAN family member between human and mice (Edelstein and Collins, 2005); they even have the same amino acid sequences in the DNA binding domain. Zfp191 mRNA is widely expressed during embryonic development and in adult tissues, suggesting it may be involved in development and basic cellular processes (Prost et al., 1999). Examination of the Zfp $191^{-/}$mouse demonstrated that this protein is indispensable for early embryonic development; Zfp $191^{-/}$mice died before embryonic day 7.5 (Li et al., 2006). ZNF24 was first noticed for its capacity to bind to a tandem repeat TCAT sequence in the first intron of human tyrosine hydroxylase $(\mathrm{TH})$ and to negatively regulate its transcription (Albanese et al., 2001). Further study suggested that the minimal repeat number of the TCAT motifs is three for ZNF24 binding, and that any point mutant in the TCAT motif can destroy this interaction (Wang et al., 2008). The transcriptional repression activity of ZNF24 has also been demonstrated in an artificial GAL4 promoter system; meanwhile. ZNF24 is involved in negative regulation of VEGF (Han et al., 1999; Harper et al., 2007). More recently, ZNF24 was found to be a pleiotropic factor involved in angiogenesis, brain development, the DNA damage response ( $\mathrm{Li}$ et al., 2009), the promotion of the cell cycle and maintenance of the progenitor stage of human neural progenitors (Khalfallah et al., 2009). However, the exact role of ZNF24/ Zfp191 in these processes remains unclear. Furthermore, as a widely expressed transcriptional factor, ZNF24/Zfp191 may be involved in additional physiological and pathologic processes.

In the present study, we first analyzed the putative binding sites of mouse Zfp191 in the promoter region of multiple genes to predict its target genes in silico. Pathway analyses of the potential target genes indicated that Zfp191 may also be involved in the immune response. This was verified in Zfp191 heterozygous knockout mice $\left(\mathrm{Zfp} 191^{+/}\right)$using a model of LPS-induced endotoxic shock, a well-established experimental model of Gram-negative bacterial infection-induced innate immunity (Remick and Ward, 2005; Mannel, 2007). These results demonstrated that the Zfp $191^{+/-}$mice were resistant to LPS-induced lethal shock due to reduced IL-1ß and IL-6 expression compared to wild-type (WT) mice. These data suggest that Zfp191 functions as a novel mediator of the innate immune response by regulating the level of the inflammatory cytokines IL-1 $\beta$ and IL-6.

\section{MATERIAL AND METHODS}

\section{Development of site search and pathway analysis}

The mouse promoter data used for analysis of the (TCAT) $)_{3}$ and the frequency of simi- 
lar sequences were obtained from the PromoSer site (http://biowulf.bu.edu/zlab/PromoSer/) (Halees et al., 2003). A search for (TCAT) $)_{3}$ and similar sequences in the promoter region of a gene was performed using the Tblast program. The frequency of related sequences was calculated using a Matlab script. Genome-wide identification of Zfp191 putative target genes was performed using the website, http://www.sitesearch.mshri.on.ca/Genome/index.html (Silvestri et al., 2008). The pathway analysis of putative target genes was performed using Pathway-Express from the Intelligent Systems and Bioinformatics Laboratory site (http://vortex. cs.wayne.edu/index.htm) (Tarca et al., 2009).

\section{Mice}

Zfp191 $11^{+-}$mice were provided by Prof. Jiliang Fu (Li et al., 2006). Zfp $191^{+/}$were backcrossed more than 10 times to the C57BL/6J genetic background. All mice were housed in a pathogen-free facility. In all experiments, heterozygous mice were compared to their wild-type littermates. All of animal studies conducted were reviewed and approved by the Institutional Animal Care and Use Committee.

\section{LPS-induced septic shock model}

Mice (8-12 weeks old) were injected intraperitoneally (ip) with $30 \mathrm{mg} / \mathrm{kg}$ body weight of Escherichia coli lipopolysaccharide (LPS, Sigma-Aldrich). Survival was monitored every $6 \mathrm{~h}$, up to $168 \mathrm{~h}$ post-infection.

\section{RT-PCR analysis}

Total RNA was isolated using TRIzol (Invitrogen). Reverse transcription was performed using random primers and M-MLV reverse transcriptase (Promega) according to manufacturer instructions. Quantitative PCR analyses were performed using an ABI 7000 TaqMan system (Applied Biosystems). The sequences of the primers were as follows: mouse actin, 5'-TACCCAGGCATTGCTGACAGG-3' (forward), 5'-ACTTGCGGTGCAC GATGGA-3' (reverse); mouse Zfp191, 5'-TCAAATGGGCATCCTGGGAAC-3' (forward), 5'-AGCTGGGGAACACCTATGTTG-3' (reverse).

\section{Histology}

Portions of the lung, liver and kidney were fixed in 4\% paraformaldehyde for $24 \mathrm{~h}$ and embedded in paraffin. The samples were sectioned, stained with H\&E and examined using light microscopy.

\section{ELISA}

Peripheral blood samples were collected from mice at the indicated times following LPS injection for isolation of sera, which were stored at $-70^{\circ} \mathrm{C}$ until analysis. The serum levels of TNF- $\alpha$, IL-1 $\beta$, IL- 6 and IL-12p70 were measured using commercially available murine ELISA kits (R\&D Systems). 


\section{Statistical analysis}

Data are reported as means $\pm \mathrm{SD}$. The statistical difference between groups was analyzed using the Student $t$-test and $\mathrm{P}<0.05$ was considered to be significant. Survival data were evaluated using a log-rank test.

\section{RESULTS}

\section{Identification of putative binding sites for Zfp191 and classification of potential target genes}

We first examined the frequency of (TCAT) $)_{3}$ sequences, and similar motifs within the promoter region, -3000 to +1000 up to and down from the transcription site within the mouse genome. Two thousands genes were randomly selected and, using the TBLAST program and allowing for a maximum of four nucleotide mismatches, we searched the promoter region of each gene for sequences homologous to (TCAT). We identified TCTTTCTTTCTT and TCATTCATTCAT as the two sequences with the highest frequency of the 2000 genes analyzed (Figure 1A). The frequency of both sequences within the promoter region was considerably higher than that expected by chance alone (206 and 103 vs 2, respectively), suggesting that these sequences functioned in the regulation of gene expression. In a previous study, we had demonstrated that the (TCTT), sequence was not recognized by the DNA binding domain of Zfp191 (Wang et al., 2008) and the (TCAT), sequence was considered as the focus of further research regarding the target gene of Zfp191. A genome-wide association scan for $(\mathrm{TCAT})_{3}$ motifs within the gene promoter region, between -2000 to +1000 from the transcription start site, which was denoted as +1 , was performed. The total number of (TCAT) motifs identified was 929, which included 355 gene promoter regions. To gain insight into the biological process in which Zfp191 was involved, we conducted a pathway analysis of the putative target genes using an online tool, Pathway-express, which can perform a search and build a list of all associated pathways involving our potential target genes (Tarca et al., 2009). The top ten pathways identified, arranged in descending order according to their biological impact, are listed (Figure 1B). Among these ten pathways, three were involved in the immune response, specifically in the toll-like receptor (TLR) signaling pathway, a cytokine-cytokine receptor pathway, and the Fc\&RI signaling pathway. A role for Zfp191 in the immune response has not been previously demonstrated. Therefore, we used a LPS-induced endotoxic shock model, which mimics a Gram-negative bacterial infection-induced innate immune response, to evaluate the role of Zfp191 in the innate immune response in vivo.

\section{Zfp191 $^{+/-}$mice have increased survival during LPS-induced endotoxic shock}

We challenged WT C57BL/6J mice with $30 \mathrm{mg} / \mathrm{kg}$ body weight of LPS and measured Zfp191 gene expression in the liver and spleen at different time points. Quantitative RT-PCR demonstrated that, in both the liver and spleen, the expression of Zfp191 was downregulated by approximately $50 \% 1.5 \mathrm{~h}$ after mice were challenged with LPS compared to the control animals (Figure 2). This showed that the expression of Zfp191 was rapidly regulated in response to LPS in mice. 

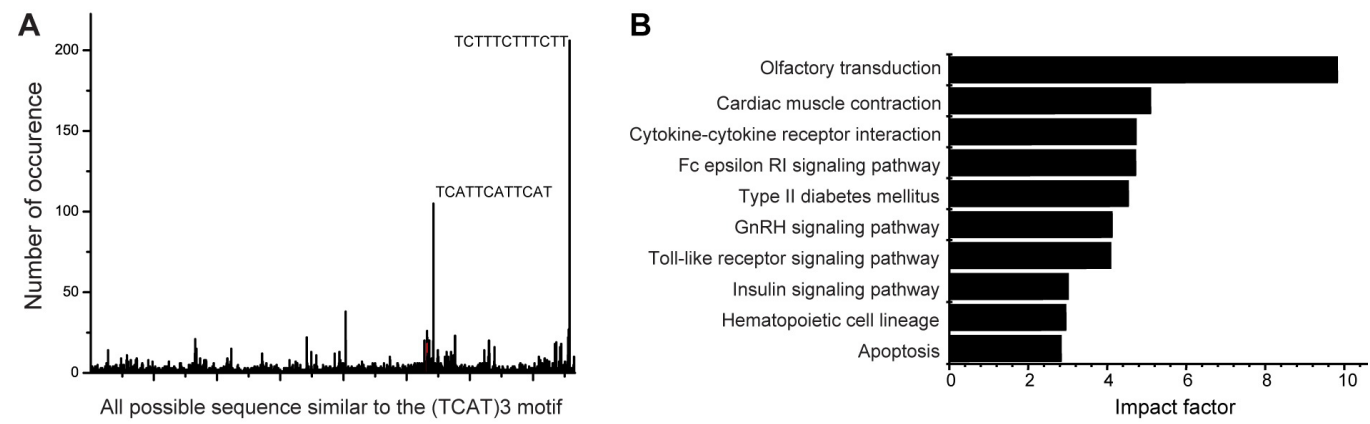

Figure 1. Bioinformatics analyses of the potential binding sites of Zfp191 and the implicated signaling pathways of the putative target genes. A. The number of all possible sequences similar to the (TCAT) $)_{3}$ motif. B. The list of pathways associated with the putative target genes, identified using Pathway-Express. The identified sequence was dependent on the impact factor given by the Pathway-Express.
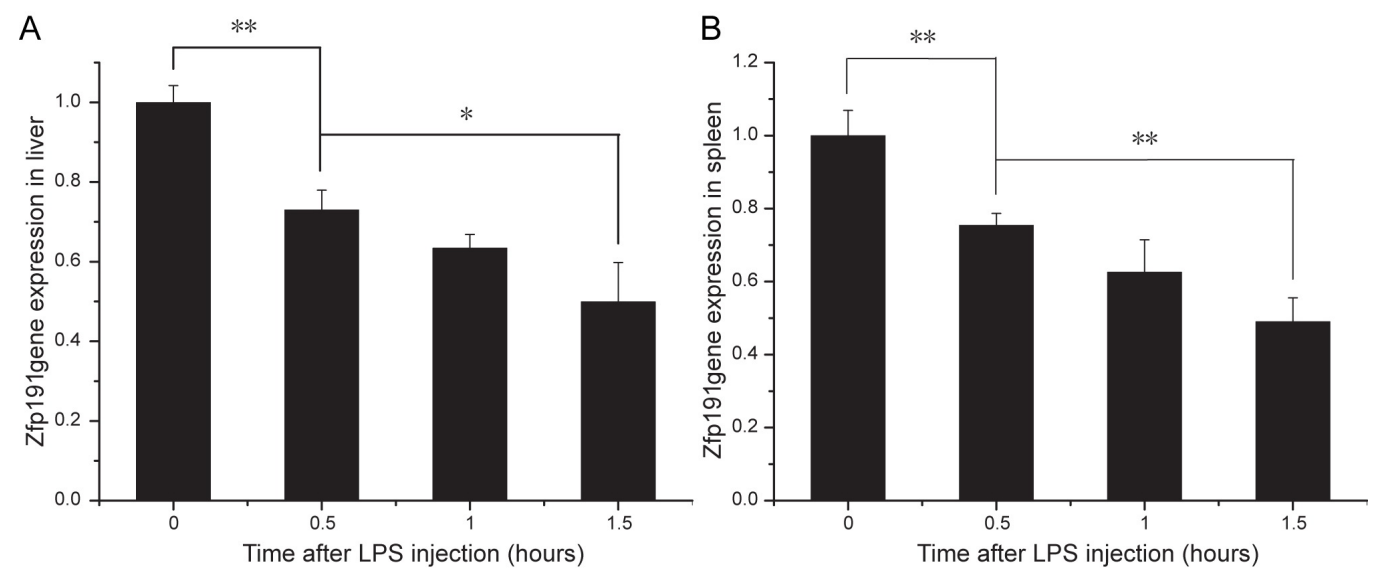

Figure 2. RT-PCR analysis of $Z f p 191$ gene expression levels after LPS injection in the liver and spleen at indicated time points. $* \mathrm{P}<0.05, * * \mathrm{P}<0.01(\mathrm{~N}=3)$.

To further elucidate the role of Zfp191 in the immune response to LPS, Zfp191 ${ }^{+/}$mice and WT littermates were also injected ip with $30 \mathrm{mg} / \mathrm{kg}$ body weight of LPS, and survival was monitored for seven days. The survival rate of $\mathrm{Zfp} 191^{+/-}$mice was significantly higher compared to WT mice; $61 \%$ of the Zfp $191^{+/-}$mice (11 of 18 mice) survived for seven days compared to $27 \%$ of wild-type mice (5 of 18 mice) (Figure 3A). This experiment was repeated three times with the same result.

\section{Reduced LPS-induced organ injury in the $\mathrm{Zfp}_{191^{+/}}$mice}

To evaluate the level of organ injury induced by LPS, the serum levels of aspartate aminotransferase (AST) and urea nitrogen (BUN), markers of liver and kidney injury, were measured $24 \mathrm{~h}$ after LPS injection (30 mg/kg, Figure 3B). The level of AST in Zfp191 ${ }^{+/}$mice 
was significantly decreased by $24.4 \%$ relative to the WT level. However, the BUN level did not differ significantly between the two groups. Histological examination of the liver, kidney and lung was also performed (Figure 3C). Consistent with the AST and BUN data, WT mice displayed a greater degree of hepatomegaly than did the $\mathrm{Zfp} 191^{+/}$animals. Though there was no significant difference in kidney injury between the two groups of mice, accumulation of red blood cells in the glomeruli was detected in both genotypes. Compared with the Zfp $191^{+-}$ mice, the lungs from WT mice displayed a greater degree of alveolar wall thickening and more severe capillary hyperemia. Taken together, these data demonstrated that the severity of LPS-induced liver and lung injury was alleviated in the $\mathrm{Zfp} 191^{+/}$mice compared to their WT littermates.
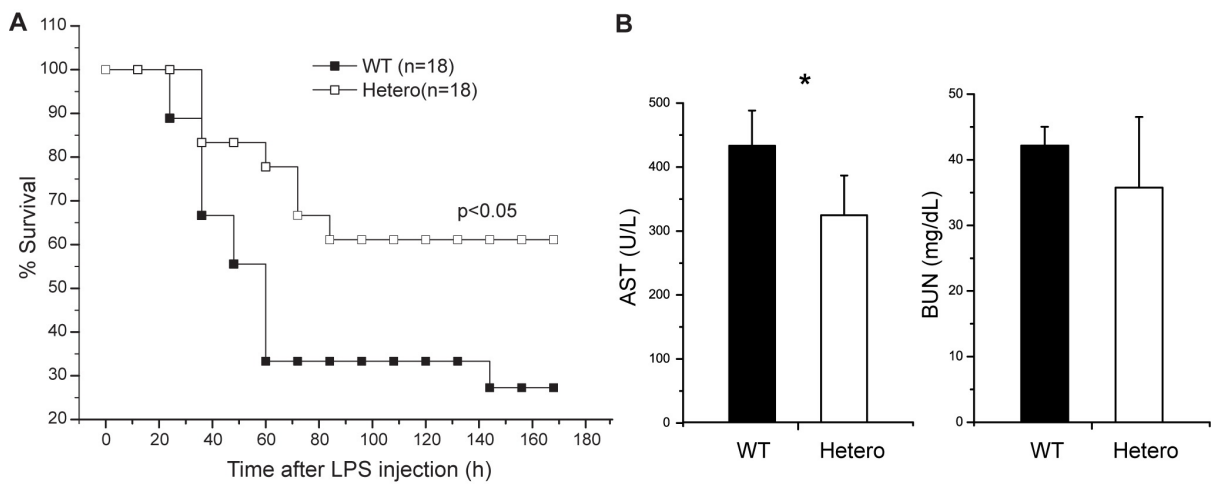

C

Liver

Kidney

Lung
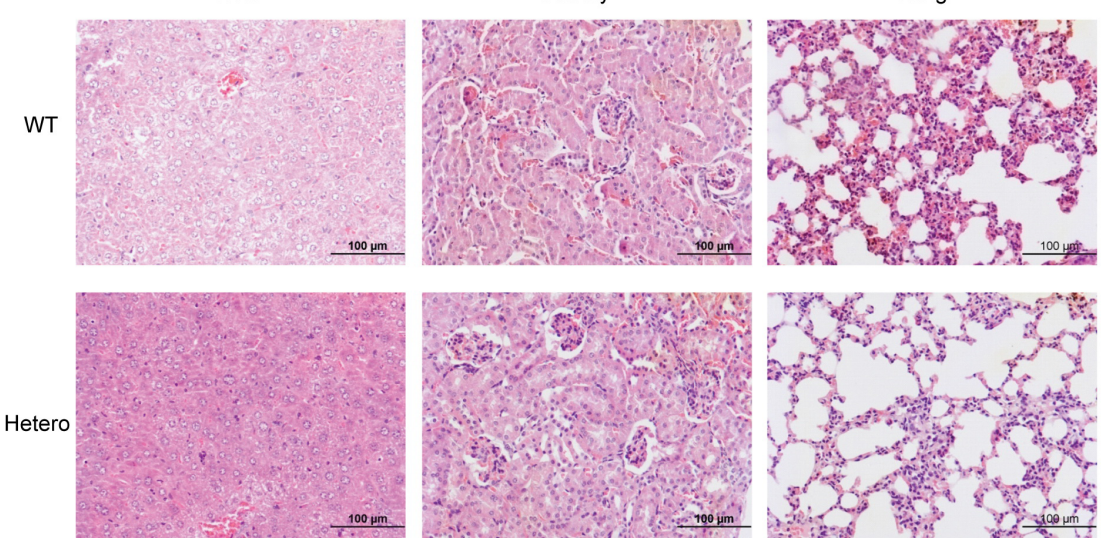

Figure 3. Zfp $191^{+/-}$mice were resistant to LPS-induced endotoxic shock at a dose of $30 \mathrm{mg} / \mathrm{kg}$ body weight. A. Survival was monitored up to $168 \mathrm{~h}$ post-LPS injection. B. AST and BUN levels in mice $24 \mathrm{~h}$ after LPS injection $(\mathrm{N}=7) .{ }^{*} \mathrm{P}<0.05$. C. Histological analysis of the H\&E stained sections of the liver, kidney and lung from WT and $\mathrm{Zfp} 191^{+/-}$mice $24 \mathrm{~h}$ after LPS injection. Magnification $=200 \mathrm{X}$.

\section{LPS-induced expression of IL-1 $\beta$ and IL-6 is reduced in $\mathrm{Zfp} 191^{+/-}$mice}

To understand the cause of the decreased susceptibility to LPS-induced septic shock seen in $\mathrm{Zfp} 191^{+/-}$mice, we measured the serum level of inflammatory cytokines at differ- 
ent time points after LPS injection (Figure 4). As critical mediators of endotoxic shock and molecular markers of sepsis, TNF- $\alpha$, IL- $1 \beta$ and IL- 6 can be induced by LPS administration in both genotypes. However, all three cytokines were reduced in $\mathrm{Zfp} 191^{+/-}$mice compared to WT animals. TNF- $\alpha$ was reduced by $25 \%$ at $1.5 \mathrm{~h}$ and $6 \mathrm{~h}$ in the Zfp $191^{+/-}$animals compared to WT littermates, although the difference was not statistically significant. This may be due to the variation from mouse to mouse. In contrast, compared to the WT mice, the serum levels of IL-1 $\beta$ were significantly reduced by 39 and $37 \%$ in $\mathrm{Zfp} 191^{+/-}$mice at $6 \mathrm{~h}$ and $24 \mathrm{~h}$, respectively. Additionally, IL-6 was also significantly reduced by 23,29 and $29 \%$ in $\mathrm{Zfp} 191^{+/-}$mice at 1.5 $\mathrm{h}, 6 \mathrm{~h}$ and $24 \mathrm{~h}$, respectively, compared to WT serum levels. IL-12p70 was also evaluated at each time point but it did not differ significantly between the two genotypes at any time point.
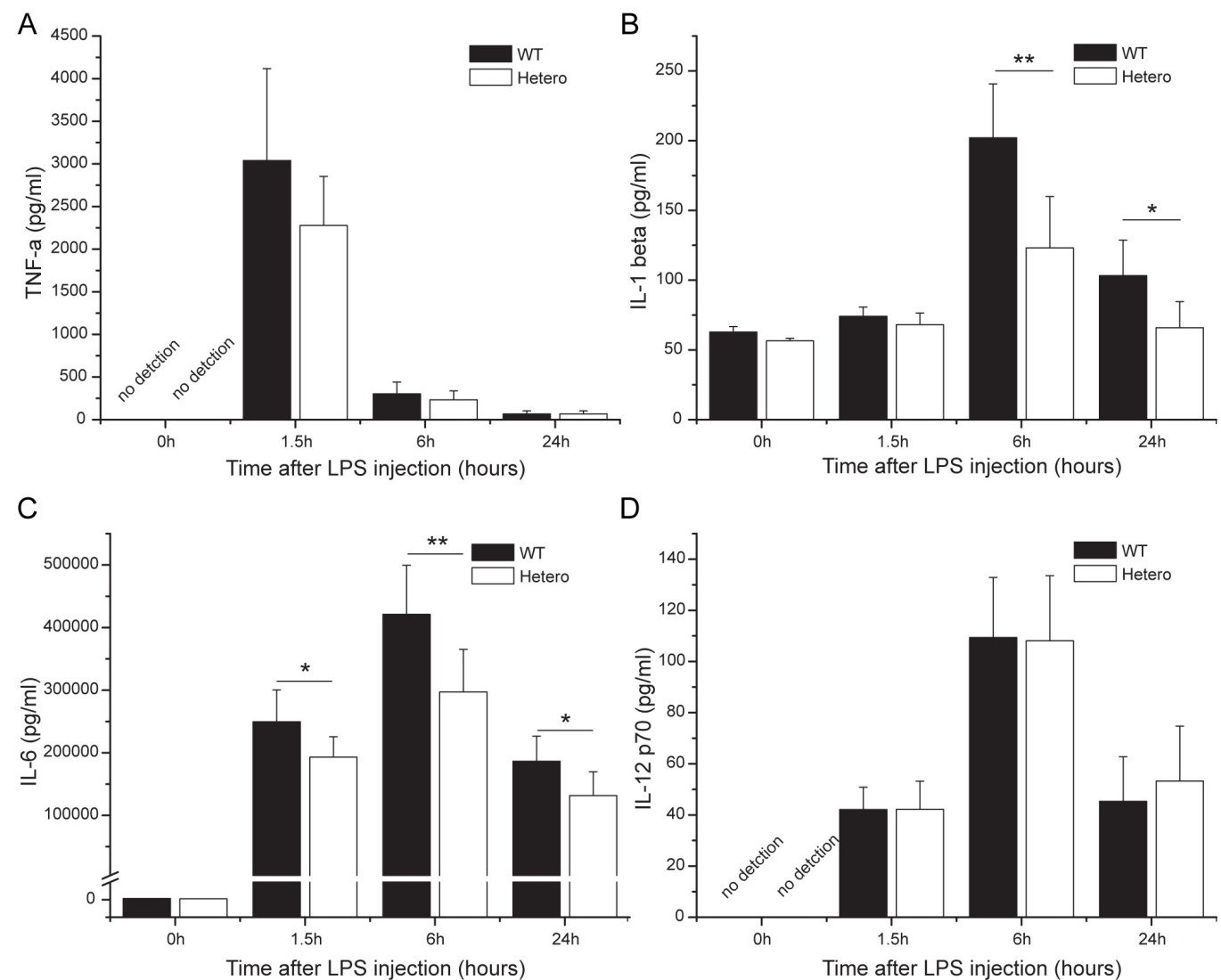

Figure 4. Serum cytokine levels after LPS injection in the Zfp $191^{+/-}$and WT mice. Serum was collected at the indicated time point and analyzed by ELISA for TNF- $\alpha$, IL-1ß, IL- 6 and IL-12p70.* $\mathrm{P}<0.05, * *, \mathrm{P}<0.01(\mathrm{~N}=4$, at $0 \mathrm{~h}$ in each group; $\mathrm{N}=6-7$, at 1.5, 6 and $24 \mathrm{~h}$ in each group).

\section{DISCUSSION}

In the current study, we utilized in silico methods to identify the possible gene targets of Zfp191 in mice according to the presence of the (TCAT) $)_{3}$ motif in their promoter region. 
Further bioinformatics analyses of these target genes suggested that a group of them are involved in the immune response. This prediction was verified in $\mathrm{Zfp} 191^{+/}$and WT mice subjected to LPS-induced septic shock. Zfp191 ${ }^{+/}$mice were found to be resistant to LPS-induced death due to reduced serum levels of IL-1 $\beta$ and IL-6 following LPS injection. Our findings are valuable for understanding the role of Zfp191 in various biological processes and reveal a new regulatory mechanism in innate immunity.

In silico methods to predict the transcriptional network have become popular and feasible with the accumulation of genome sequence data. In the current study, 929 putative binding sites for Zfp191were identified, which were localized in the promoter region of 355 genes. These genes were enriched for 55 associated pathways and the olfactory transduction pathway had the highest impact factor, implicating Zfp191 in the process of odorant sensing. Furthermore, Zfp191 transcripts were highly expressed in the olfactory placode and in the epithelium of the nose during mouse embryogenesis (Khalfallah et al., 2008) and were present at a high level in the olfactory bulb, which was in contrast to the low level detected in the entire rat brain (Albanese et al., 2001). Hematopoietic cell lineage and apoptosis pathways, nine and ten on the prediction list, were also in agreement with a previous study demonstrating that ZNF24 has a role in hematopoiesis and in response to cellular DNA damage (Noll et al., 2008; Li et al., 2009). Notably, among the top 10 pathways listed, three are related to the immune response: a cytokine-cytokine receptor interaction pathway, the FceRI signaling pathway and the TLR signaling pathway. The cytokine-cytokine receptor interaction pathway plays an important role in the innate and adaptive inflammatory response, in which cytokines are released and mobilize immune cells to respond to the stimulus by interaction with receptors. The FceRI signaling pathway is a key pathway in the development of allergic inflammatory reactions, which are initiated by the interaction of an antigen with $\operatorname{IgE}$ bound to the $\alpha$-chain of FceRI and, ultimately, results in the release of various mediators of allergic inflammation and anaphylactic reactions (Roth et al., 2008). The TLR signaling pathway plays an essential role in detecting microbial pathogens and generating an innate immune response. A role for Zfp191 in the immune response has not been previously shown. In our study, the prediction of Zfp191 involvement in immune function was verified using a LPS-induced endotoxic shock model in the Zfp $191^{+/-}$mice. Collectively, the data provide evidence, in addition to the studies mentioned above, that in silico methods can be used to predict the physiological function of Zfp191 in vivo.

In the LPS-induced endotoxic shock model, LPS is recognized by TLR4 expressed on innate immune cells, provoking rapid activation of innate immunity via the upregulation of proinflammatory cytokines and co-stimulatory molecules, such as TNF- $\alpha$, IL-1, IL-6, COX-2, and iNOS (Manthey et al., 1998; Lee et al., 1999; Kyriakis and Avruch, 2001). Zfp191 ${ }^{+/}$mice had reduced serum levels of IL-1ß and IL-6 in response to LPS-induced endotoxic shock. IL-1 and IL-6 are important pro-inflammatory cytokines that increase in both clinical and experimental (mouse) sepsis (Sriskandan and Altmann, 2008). IL-1 exerts a profound effect on the vasculature and endothelium, promoting marked vasodilatation, which is associated with vascular leakage (Sriskandan and Altmann, 2008). It is also a powerful coagulant that can promote fibrin clot deposition in small blood vessels (van der Poll and van Deventer, 1999), leading to tissue hypoperfusion and organ failure. IL-6 is a pleiotropic cytokine that can promote inflammation through the expansion and activation of T cells and the differentiation of B cells (Moriyama et al., 2006; Sriskandan and Altmann, 2008). Most importantly, because there is a 
correlation between increase in serum IL-6 and mortality, it may be an important prognostic marker for sepsis and septic shock (Watanabe et al., 2005). Thus, the low serum level of IL-1 and IL-6 may explain the enhanced survival of Zfp191 ${ }^{+-}$mice after LPS-induced endotoxic shock compared to WT littermates.

Although we demonstrated that Zfp191 was involved in LPS-induced endotoxic shock, its direct target genes and regulation mechanisms mediating LPS-induced endotoxic shock remain unclear. Further study on the regulatory mechanisms of Zfp191, based on the identification of target genes in LPS-induced endotoxic shock, is necessary.

In conclusion, we report that Zfp191 was involved in LPS-induced endotoxic shock and that the deletion of one copy of the Zfp191 gene promoted survival of LPS-treated mice via the reduction of IL-1 $\beta$ and IL-6. These findings suggest that Zfp191 may function as a novel immune mediator, and that the loss of Zfp191 can suppress the systemic inflammatory response by reducing cytokine production.

\section{ACKNOWLEDGMENTS}

Research supported by the National 863 High-Tech Project (\#2008AA02Z126), the Science and Technology Commission of Shanghai Municipality (\#54319939, \#06XD14014, and \#07DZ19503) and the E-Institutes of Shanghai Municipal Education Commission (project \#E03003). We thank Jiajuan Shen for generating the antibodies.

\section{REFERENCES}

Albanese V, Biguet NF, Kiefer H, Bayard E, et al. (2001). Quantitative effects on gene silencing by allelic variation at a tetranucleotide microsatellite. Hum. Mol. Genet. 10: 1785-1792.

Edelstein LC and Collins T (2005). The SCAN domain family of zinc finger transcription factors. Gene 359: 1-17.

Halees AS, Leyfer D and Weng Z (2003). PromoSer: A large-scale mammalian promoter and transcription start site identification service. Nucleic Acids Res. 31: 3554-3559.

Han ZG, Zhang QH, Ye M, Kan LX, et al. (1999). Molecular cloning of six novel Kruppel-like zinc finger genes from hematopoietic cells and identification of a novel transregulatory domain KRNB. J. Biol. Chem. 274: 35741-35748.

Harper J, Yan L, Loureiro RM, Wu I, et al. (2007). Repression of vascular endothelial growth factor expression by the zinc finger transcription factor ZNF24. Cancer Res. 67: 8736-8741.

Khalfallah O, Faucon-Biguet N, Nardelli J, Meloni R, et al. (2008). Expression of the transcription factor Zfp191 during embryonic development in the mouse. Gene Expr. Patterns 8: 148-154.

Khalfallah O, Ravassard P, Lagache CS, Fligny C, et al. (2009). Zinc finger protein 191 (ZNF191/Zfp191) is necessary to maintain neural cells as cycling progenitors. Stem Cells 27: 1643-1653.

Kyriakis JM and Avruch J (2001). Mammalian mitogen-activated protein kinase signal transduction pathways activated by stress and inflammation. Physiol. Rev. 81: 807-869.

Lee JC, Kassis S, Kumar S, Badger A, et al. (1999). p38 mitogen-activated protein kinase inhibitors-mechanisms and therapeutic potentials. Pharmacol. Ther. 82: 389-397.

Li J, Chen X, Yang H, Wang S, et al. (2006). The zinc finger transcription factor 191 is required for early embryonic development and cell proliferation. Exp. Cell Res. 312: 3990-3998.

Li J, Chen X, Gong X, Liu Y, et al. (2009). A transcript profiling approach reveals the zinc finger transcription factor ZNF191 is a pleiotropic factor. BMC Genomics 10: 241.

$\mathrm{Lu}$ D, Searles MA and Klug A (2003). Crystal structure of a zinc-finger-RNA complex reveals two modes of molecular recognition. Nature 426: 96-100.

Mannel DN (2007). Advances in sepsis research derived from animal models. Int. J. Med. Microbiol. 297: 393-400.

Manthey CL, Wang SW, Kinney SD and Yao Z (1998). SB202190, a selective inhibitor of p38 mitogen-activated protein kinase, is a powerful regulator of LPS-induced mRNAs in monocytes. J. Leukoc. Biol. 64: 409-417.

Moriyama M, Matsukawa A, Kudoh S, Takahashi T, et al. (2006). The neuropeptide neuromedin U promotes IL-6 
production from macrophages and endotoxin shock. Biochem. Biophys. Res. Commun. 341: 1149-1154.

Noll L, Peterson FC, Hayes PL, Volkman BF, et al. (2008). Heterodimer formation of the myeloid zinc finger 1 SCAN domain and association with promyelocytic leukemia nuclear bodies. Leuk. Res. 32: 1582-1592.

Prost JF, Negre D, Cornet-Javaux F, Cortay JC, et al. (1999). Isolation, cloning, and expression of a new murine zinc finger encoding gene. Biochim. Biophys. Acta 1447: 278-283.

Remick DG and Ward PA (2005). Evaluation of endotoxin models for the study of sepsis. Shock 24 (Suppl 1): 7-11.

Roth K, Chen WM and Lin TJ (2008). Positive and negative regulatory mechanisms in high-affinity IgE receptor-mediated mast cell activation. Arch. Immunol. Ther. Exp. 56: 385-399.

Silvestri C, Narimatsu M, von B, I, Liu Y, et al. (2008). Genome-wide identification of Smad/Foxh1 targets reveals a role for Foxh1 in retinoic acid regulation and forebrain development. Dev. Cell 14: 411-423.

Sriskandan S and Altmann DM (2008). The immunology of sepsis. J. Pathol. 214: 211-223.

Tarca AL, Draghici S, Khatri P, Hassan SS, et al. (2009). A novel signaling pathway impact analysis. Bioinformatics 25: 75-82.

van der Poll T and van Deventer SJ (1999). Cytokines and anticytokines in the pathogenesis of sepsis. Infect. Dis. Clin. North Am. 13: 413-26, ix.

Wang H, Sun R, Liu G, Yao M, et al. (2008). Characterization of the target DNA sequence for the DNA-binding domain of zinc finger protein 191. Acta Biochim. Biophys. Sin. 40: 704-710.

Watanabe E, Hirasawa H, Oda S, Matsuda K, et al. (2005). Extremely high interleukin-6 blood levels and outcome in the critically ill are associated with tumor necrosis factor- and interleukin-1-related gene polymorphisms. Crit. Care Med. 33: 89-97. 